\title{
Trabecular Plate Loss and Deteriorating Elastic Modulus of Femoral Trabecular Bone in Intertrochanteric Hip Fractures
}

Ji Wang'1, Bin Zhou', Ian Parkinson², C. David L. Thomas³, John G. Clement³, Nick Fazzalari², X. Edward $\mathrm{Guo}^{1 *}$

${ }^{1}$ Bone Bioengineering Laboratory, Department of Biomedical Engineering, Columbia University, New York, New York 10027, USA; 'Bone and Joint Research Lab, SA Pathology and Discipline of Anatomy and Pathology, University of Adelaide, Adelaide, Australia; ${ }^{3 S e c t i o n}$ of Oral Anatomy and Surgery, The Melbourne Dental School, University of Melbourne, Victoria 3010, Australia

Osteoporotic hip fracture is associated with significant trabecular bone loss, which is typically characterized as low bone density by dual-energy X-ray absorptiometry (DXA) and altered microstructure by micro-computed tomography $(\mu \mathrm{CT})$. Emerging morphological analysis techniques, e.g. individual trabecula segmentation (ITS), can provide additional insights into changes in plate-like and rod-like trabeculae, two major microstructural types serving different roles in determining bone strength. Using ITS, we evaluated trabecular microstructure of intertrochanteric bone cores obtained from 23 patients undergoing hip replacement surgery for intertrochanteric fracture and 22 cadaveric controls. Micro-finite element ( $\mu \mathrm{FE})$ analyses were performed to further understand how the abnormalities seen by ITS might translate into effects on bone strength. ITS analyses revealed that, near fracture site, plate-like trabeculae were seriously depleted in fracture patients, but trabecular rod volume was maintained. Besides, decreased plate area and rod length were observed in fracture patients. Fracture patients also showed decreased elastic moduli and shear moduli of trabecular bone. These results provided evidence that in intertrochanteric hip fracture, preferential loss of plate-like trabeculae led to more rod-like microstructure and deteriorated mechanical competence adjacent to the fracture site, which increased our understanding of the biomechanical pathogenesis of hip fracture in osteoporosis. Keywords: hip fracture; intertrochanteric; microstructure; individual trabecula segmentation; finite element

Bone Research (2013) 4: 346-354. doi: 10.4248/BR201304005

\section{Introduction}

Hip fracture in the elderly has been an increasingly serious threat to public health (1) and the most devastating outcome of osteoporosis, resulting in high morbidity, mortality and healthcare expense (2). The incidence of hip fracture is projected to double in the coming decades

*Correspondence: X. Edward Guo

E-mail: ed.guo@columbia.edu

Tel: 212854 6196; Fax: 2128548725

Received 27 August 2013; Accepted 21 October 2013 due to unprecedented global population aging $(1,3)$. Despite the established relationship between increased risk of hip fracture and decreased areal bone mineral density (aBMD), over half of the postmenopausal women who have sustained a hip fracture have aBMD higher than the WHO threshold for osteoporosis (4-5). These facts underlie the need to understand other risk factors for osteoporotic hip fracture, such as deteriorating trabecular bone microstructure and increasing bone fragility associated with a ging and osteoporosis.

Hip fracture occurs most often in the femoral neck and intertrochanteric region, where trabecular bone contri- 
butes significantly to whole bone mechanical integrity and experiences dramatic mic rostructural changes during aging. Greater age-related bone loss was observed in trabecular volumetric bone mineral density (vBMD) than that of cortical bone, as measured by quantita tive computed tomography (QCT) at the hip (6). Most studies regarding hip fracture risk focused on femoral neck subregions. For example, the posterior femoral neck tended to show the most prominent stuctural changes associated with aging (7-8). Recently, C arballido-Ga mio et al. (9) discovered that bone loss in hip fracture patients occurred not only at femoral neck but also expanded to the intertrochanteric trabecularbone region, which was ra rely studied. Therefore, it is of clinic al importance to investigate microstructural and mechanical alterations of the intertrochanteric trabecular bone in patients with hip fractures compared to age-matched nonfracture subjects.

Several studies reported lower trabecular bone volume fraction, lower connectivity, more rod-like trabeculae, and higherdegree of anisotropy in patients with femoral neck fractures compared to healthy controls (7, 10-11). Furthermore, femoral neck trabecular bone biopsies from fracture patients showed lower modulus and ultimate stress as measured by mechanical testing experiments (10). A more recent study examined the microstructural differences in femoral neck trabecular bone between hip fracture patients and controls, and confimed signific ant decrease in bone volume fraction and a transition from plate-like to rod-like trabeculae in hip fracture patients (12). However, mic rostructural and biomechanical changes associated with intertrochanteric fractures have not been studied, and cannot be derived from the previous femoral neck studies due to heterogeneous trabecular bone distribution in the proximal femur (13-14). Also, Li et al. suggested that location of hip fracture was most strongly associated with the vBMD near the site of fractures in femoral neck and intertrochanteric region (15). Thus, region-specific measurements near the fracture site are needed to study trabecular bone mic rostructure in intertrochanteric hip fractures.

As suggested by previous studies, there is a significant change from plate-like to rod-like trabeculae in aging and osteoporosis $(12,16)$. However, quantific ation of the amount of trabecular plates or rods was limited. We developed individual trabecula segmentation (ITS)based monhological analysis, which resolved threedimensional (3D) trabecular bone image into individual trabeculae and provided explic it morphological evaluation for trabecular plates and rods. We have shown that trabecular plates and rods of different orientations had distinct roles in mechanical properties and failure mecha nisms of tra becular bone (17-19). Also, ITS a na lysis has revealed the key differences between Caucasian American and Chinese American skeleton, demonstrating that higher plate/rod ratio accounted for reduced fracture risk in Chinese Americ ans in spite of similar aBMD (20). In a ddition, ITS and mic ro finite element ( $\mu \mathrm{FE}$ ) a na lyses were able to distinguish postmenopausal women with and without fragility fractures independent of aBMD (12).

In this study, we compared the ITS measurements of intertrochanteric trabecular microstructure and $\mu \mathrm{FE}$ measurements of bone mechanic al properties between individuals with and without intertrochanteric hip fractures. We hypothesized that intertrochanteric fracture was associated with reduced trabecular plate volume, more rod-like microstucture and decreased trabecular bone elastic modulus.

\section{Materials and Methods}

\section{Specimen preparation}

Twenty-three subjects $(80 \pm 11$ years old) with intertrochanteric hip fractures were included in this study, consisting of 14 females and 9 males. Trabecularbone cores (10 $\mathrm{mm}$ diameter, up to $30 \mathrm{~mm}$ long) were obtained adjacent to the fracture site in intertrochanteric region during hip-replacement surgery, with rotation of the coring device into the shaft of the femur. The longitudinal axis of the surgical specimen was aligned along the cranio-caudal direction, and such orientation was maintained from specimen sampling through storage and imaging. Anothertwenty-two intertrochanteric bone cores were obtained from human cadavers of 9 female and 13 male donors ( $75+11$ years old) and used ascontrol specimens (21). The sampling position of the surgical specimensand the autopsy controls wa sequivalent. The a utopsy samples were deliberately collected ascontrols for surgery-sourced samples from patients undergoing hip replacement. Prior to autopsy, all individuals included in the control group had whole-body CT performed, which enabled exclusion of individuals with bone-related pathology. The mean age of the fracture and control groups was not statistically different $(P>0.05)$. All specimens were stored in saline-soaked gauze and frozen at $-70{ }^{\circ} \mathrm{C}$ prior to micro CTimaging.

\section{Mic ro CTimaging}

Each trabecular bone core was scanned at $15 \mu \mathrm{m}$ isotropic voxel size using a micro computer tomography $(\mu C T)$ system (Skyscan model 1174, Skyscan, Aartselaar, Belgium). A cubic sub-volume $\left(4.44 \times 4.44 \times 4.44 \mathrm{~mm}^{3}\right)$ was extracted from the center of each reconstructed $3 D$ 
image. The grayscale images were binarized using a global thresholding technique. Isolated voxels or disc onnected voxel-clusters were removed from the largest bone component, and the resulting images were used for the following analyses.

\section{Standard $\mu$ CTmorphological a nalyses}

The standard morphological parameters such as BV/TV, trabecular number (Tb.N), trabecular thic kness (Tb.Th), trabecular spacing (Tb.Sp), structure model index (SMI), bone surface to bone volume ratio (BS/BV), and degree of anisotropy (DA) were evaluated for each specimen using the standard morphological a nalysis softwa re on a Skysc an model 1174 system.

\section{ITS-based morphological a nalyses}

A complete volumetric decomposition technique was applied to segment the trabecular bone network into individual trabecular plates and rods (Figure 1). Biefly, the surface and curve skeleton of the trabecular bone microstructure was first extracted through an iterative thinning process, while preserving the topology and morphology of the original mic rostructure (22). Then, the entire skeleton was decomposed into individual plate and rod skeletons with each skeletal voxel uniquely classified as either a plate or a rod type based on digital

A

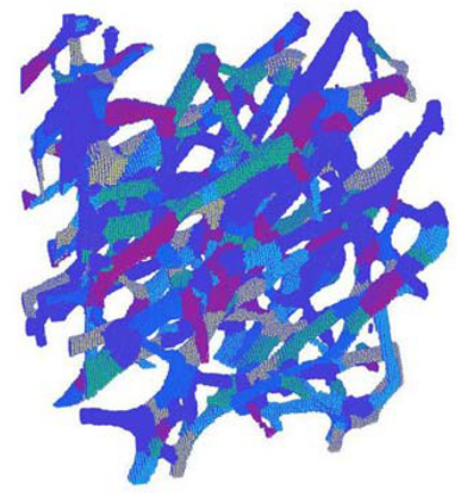

C

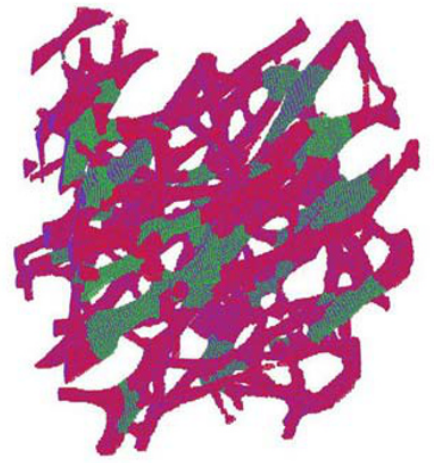

topological classification (23). Using an iterative reconstruction method, each voxel of the original image was classified as belonging to either an individual plate or rod (Figure 1) (18).

ITS-based 3D evaluation of individual tra beculae measured plate-and rod-specific morphological parameters. The plate and rod bone volume fraction ( $p B V / T V$ and rBV/TV) represented the proportion of plate and rod trabecular volume in the bulk volume. Plate and rod tissue fraction ( $p B V / B V$ and $r B V / B V$ ) were the total volume of plate and rod bone volume divided by the total bone volume. The number of plates and rods was denoted by corresponding number densities ( $p$ Tb.N and rTb.N, $\mathrm{mm}^{-1}$ ). The average size of plates and rods was quantified by plate and rod thic kness ( $p$ Tb.Th and $r \mathrm{~Tb}$.Th, $\mathrm{mm})$, plate surface area (pTb.S, $\mathrm{mm}^{2}$ ), and rod length (rTb.L, mm). Plate-to-rod ratio (P-R ratio), a parameter of plate versus rod characteristic s of trabecular bone, was defined as plate bone volume divided by rod bone volume. Trabecular network intactness was characterized by plate-plate, plate-rod, and rod-rod junction density (P-P, P-R, and R-R J unc.D, $\mathrm{mm}^{-3}$ ), calculated as the total junctions between trabecular plates and rods normalized by the bulk volume. Orientation of the trabecular bone network was characterized by axial bone volume fraction (aBV/TV), defined asaxially aligned bone

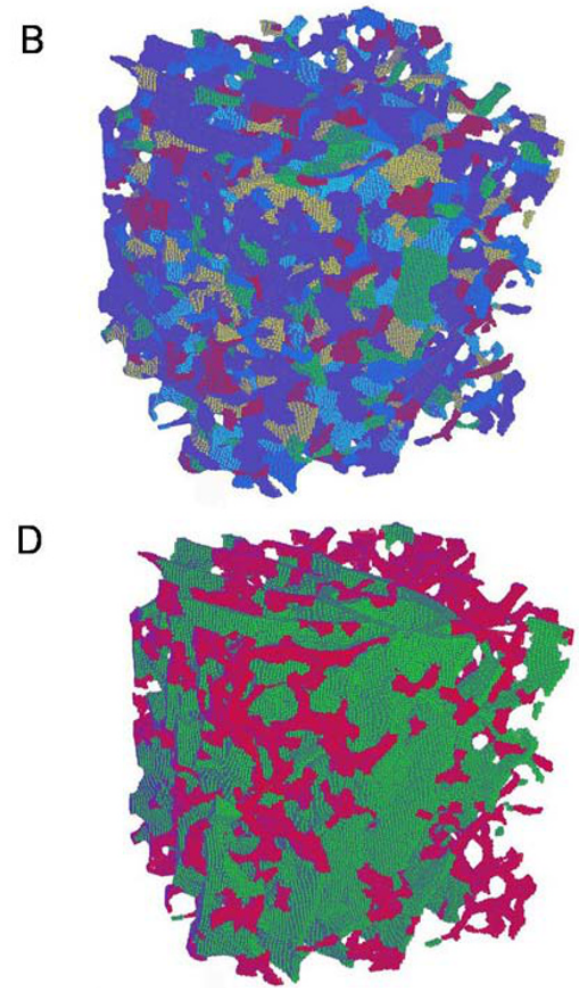

Figure 1 Fully decomposed trabecular microstructure of the fracture group (A) and the control group (B), with different colors representing individual trabeculae. Trabecular plates (green) and rods (red) were displayed for the fracture group (C) and the control group (D). 
volume divided by the bulk volume.

\section{$\mu \mathrm{FE}$ a na lyses}

A finite element (FE) model was generated from each image by converting each voxel to an eight-node brick element. The trabecular bone tissue was modeled as an isotropic, linear elastic material with a Young's modulus (Es) of $15 \mathrm{GPa}$ and a Poisson's ratio of 0.3 for all the models (24). Six FE analyses were perfomed for each model, representing three uniaxial compression tests along imaging axes ( $X, Y$ and $Z$ ) and three uniaxial shear tests (25). The $X, Y, Z$ axes were defined along the three orthogonal edges of the cubical image, among which $Z$ axis was along the longitudinal direction of the trabecularbone core and $x$ and $y$ axes were in the transverse plane. The three elastic moduli along imaging axes (EXX, EYY, EZl) and three shear moduli (GXY, GXY, GXY) were derived from the FE analyses.

Statistic al a nalyses

Statistic al a na lyses were performed using NCSS softwa re
(NCSS 2007, NCSS Statistical Software, Kaysville, Utah). Data were presented in the form of mean \pm standard deviation (SD). Student's t-test was used to determine differences in ITS mic rostructural and mechanical parameters between fracture and control groups. Two-sided $\mathrm{P} \varangle 0.05$ was considered to indic a te statistic al signific ance. Power law nonlinear regression analysis was used to examine the relation between axial elastic moduli and $\mathrm{BV} / \mathrm{TV}, \mathrm{pBV} / \mathrm{TV}$ and $\mathrm{aBV} / \mathrm{TV}$.

\section{Results}

According to the standard $\mu C T$ morphological evaluation, trabecular mic rostructure in fracture patients showed lower BV/TV, Tb.N, Tb.Th and higher Tb.Sp than the nonfracture controls. No significant difference was found in SMI, which characterized trabecular mic rostructure as being either plate-like $(S M I=0)$ or rod-like (SMI=3). However, ITS a nalyses revealed striking changes in plate- and rod-like trabeculae associated with the status of fracture (Table 1, Figure 2). On average, the

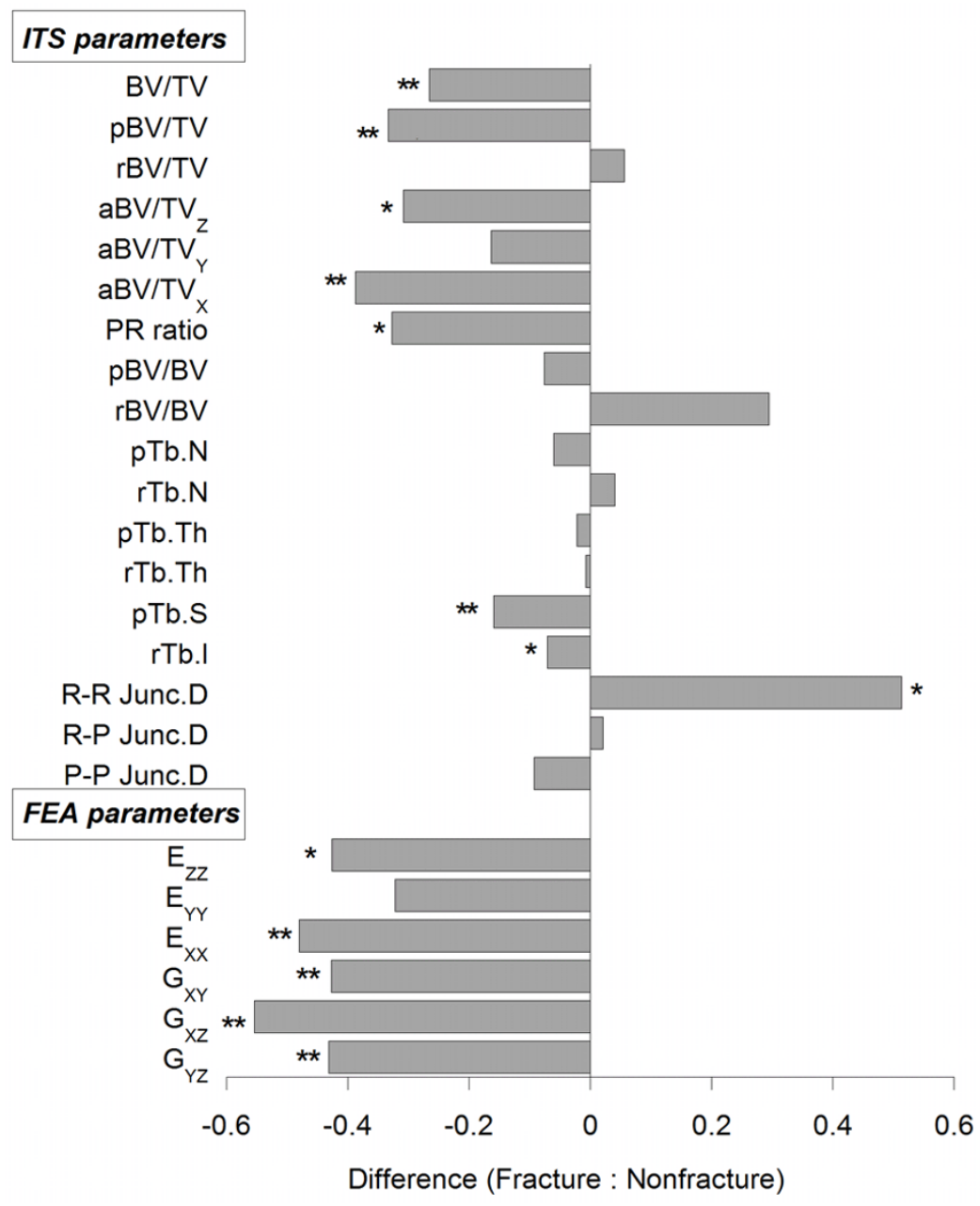

Figure 2 Fraction differences in ITS and $\mu$ FEA parameters between the fracture and control group. ${ }^{*} P<0.05,{ }^{* *} P<0.005$.

www.boneresearch.org | Bone Research 
Table 1 Comparison of standard $\mu \mathrm{CT}$ morphological, ITS and mechanical parameters in subjects with and without hip fractures $(\mathrm{a} P<0.05, \mathrm{~b} P<0.005, \mathrm{c} P<0.001)$.

\begin{tabular}{|c|c|c|c|c|}
\hline \multicolumn{2}{|c|}{ Variable (units) } & \multirow{2}{*}{$\begin{array}{r}\text { Fracture }(n=23) \\
8.520 \pm 0.550\end{array}$} & \multirow{2}{*}{$\begin{array}{c}\text { Nonfracture }(n=22) \\
11.600 \pm 0.870\end{array}$} & \multirow{2}{*}{$\frac{\text { P-value }}{0.004^{b}}$} \\
\hline$\mu \mathrm{CT}$ & BV/TV (\%) & & & \\
\hline & $\mathrm{Tb} . \mathrm{N}\left(\mathrm{mm}^{-1}\right)$ & $0.860 \pm 0.060$ & $1.090 \pm 0.090$ & $0.039^{a}$ \\
\hline & Tb.Th $(\mu \mathrm{m})$ & $154.940 \pm 2.490$ & $170.030 \pm 5.510$ & $0.015^{a}$ \\
\hline & Tb.Sp ( $\mu \mathrm{m})$ & $987.430 \pm 39.100$ & $865.350 \pm 36.120$ & $0.027 a$ \\
\hline & $\mathrm{BS} / \mathrm{BV}\left(\mathrm{mm}^{-1}\right)$ & $0.352 \pm 0.009$ & $0.359 \pm 0.008$ & 0.554 \\
\hline & DA & $2.370 \pm 0.120$ & $2.290 \pm 0.110$ & 0.600 \\
\hline & SMI & $1.770 \pm 0.080$ & $1.560 \pm 0.110$ & 0.131 \\
\hline \multirow[t]{17}{*}{ ITS } & pBV.TV-1 (\%) & $6.380 \pm 0.520$ & $9.570 \pm 0.920$ & $0.004^{b}$ \\
\hline & rBV.TV-1 (\%) & $2.110 \pm 0.170$ & $2.000 \pm 0.130$ & 0.603 \\
\hline & pBV.BV-1 (\%) & $73.340 \pm 2.290$ & $79.410 \pm 2.440$ & 0.077 \\
\hline & rBV.BV-1 (\%) & $26.660 \pm 2.290$ & $20.590 \pm 2.440$ & 0.077 \\
\hline & aBV. $\operatorname{TV}_{x^{-1}}(\%)$ & $3.250 \pm 0.360$ & $5.300 \pm 0.550$ & $0.003^{b}$ \\
\hline & a BV.TVY-1 (\%) & $3.110 \pm 0.300$ & $3.720 \pm 0.400$ & 0.227 \\
\hline & a BV. $\operatorname{TV}_{Z^{1}}(\%)$ & $4.020 \pm 0.430$ & $5.820 \pm 0.570$ & $0.015^{a}$ \\
\hline & P-R ratio & $3.640 \pm 0.500$ & $5.410 \pm 0.690$ & $0.043^{a}$ \\
\hline & pTb.N $\left(\mathrm{mm}^{-1}\right)$ & $2.180 \pm 0.060$ & $2.320 \pm 0.080$ & 0.181 \\
\hline & $\mathrm{rTb} . \mathrm{N}\left(\mathrm{mm}^{-1}\right)$ & $1.870 \pm 0.060$ & $1.800 \pm 0.040$ & 0.308 \\
\hline & pTb.Th (mm) & $0.112 \pm 0.002$ & $0.114 \pm 0.002$ & 0.391 \\
\hline & $\mathrm{rTb} . \mathrm{Th}(\mathrm{mm})$ & $0.104 \pm 0.002$ & $0.105 \pm 0.001$ & 0.741 \\
\hline & $\mathrm{pTb} . \mathrm{S}\left(\mathrm{mm}^{2}\right)$ & $0.053 \pm 0.002$ & $0.063 \pm 0.002$ & $0.001^{b}$ \\
\hline & rTb. $\ell(\mathrm{mm})$ & $0.329 \pm 0.007$ & $0.354 \pm 0.008$ & $0.027^{a}$ \\
\hline & R-R J unc.D ( $\left.\mathrm{mm}^{-3}\right)$ & $3.320 \pm 0.350$ & $2.200 \pm 0.230$ & $0.011^{a}$ \\
\hline & R-P J unc.D $\left(\mathrm{mm}^{-3}\right)$ & $12.060 \pm 1.040$ & $11.810 \pm 0.820$ & 0.855 \\
\hline & P-P J unc.D $\left(\mathrm{mm}^{-3}\right)$ & $9.870 \pm 0.870$ & $10.890 \pm 1.050$ & 0.459 \\
\hline \multirow[t]{6}{*}{$\mu \mathrm{FE}$} & Exx $(\mathrm{MPa})$ & $202.920 \pm 30.180$ & $382.840 \pm 49.410$ & $0.003^{b}$ \\
\hline & $\mathrm{E}_{Y Y}(\mathrm{MPa})$ & $186.040 \pm 20.800$ & $274.270 \pm 39.190$ & 0.050 \\
\hline & $\mathrm{E}_{\mathbb{Z}}(\mathrm{MPa})$ & $237.920 \pm 35.340$ & $414.570 \pm 54.010$ & $0.008^{a}$ \\
\hline & $\mathrm{G}_{X Y}(\mathrm{MPa})$ & $66.910 \pm 6.030$ & $116.780 \pm 13.530$ & $0.001^{b}$ \\
\hline & $\mathrm{G}_{x z}(\mathrm{MPa})$ & $86.630 \pm 11.110$ & $194.260 \pm 24.320$ & $<0.001^{c}$ \\
\hline & $\mathrm{G}_{\mathrm{YZ}}(\mathrm{MPa})$ & $82.340 \pm 9.660$ & $144.730 \pm 16.510$ & $0.002 b$ \\
\hline
\end{tabular}

$\mathrm{BV} / \mathrm{TV}$ in fracture subjects was $27 \%$ lower than that of the controls. More specifically, the pBV/TV was 33\% lower in fracture patients, while the rBV/TV was not different between the two groups. As a result, the P-R ratio was $32.4 \%$ lower in the fracture subjects. However, the number of trabecular plates (pTb.N) and rods (rTb.N) did not differ between the fracture and control groups. Significant difference was found in the size of trabecular plates and rods. The average trabecular plate surface area was $17 \%$ smaller and the trabecular rods were $7 \%$ shorter in fracture subjects, in spite of similar thickness. The R-R J unc.D was $51 \%$ higher in fracture subjects, while the P-P J unc.D. and P-R J unc.D were similar. Axial bone volume fraction along imaging axes (aBV/TVX, aBV/TVY, aBV/TVZ were $39 \%, 16 \%$ and $31 \%$ lower in the fracture subjects than the controls, indic ating that the trabecular bone network was less axially aligned along the three axes in fracture subjects. The orientation of $Z$ axis was maintained in all the surgical and autopsy specimens along the cranio-caudal direction. Alignment with $X$ and $Y$ axes indicated how much the trabecular microstructure wasaligned in the transverse plane, though the orientation of $X$ and $Y$ axes could not be determined anatomically due to the surgical coring procedure. Accordingly, axial elastic moduli along the three axes (EXX, EYY, EZZ) were $47 \%, 32 \%$ and $43 \%$ lower in fracture subjects. Shear moduli, GXY, GXZ, GYZ, were 43\%, 55\% and $43 \%$ lower in fracture subjects than the controls.

Significant correlations by power law were found between axial elastic moduli (Eii) and bone volume fraction (BV/TV), plate bone volume fraction ( $p B V / T V)$, and axial bone volume fraction (aBV/TVi) for three axial directions ( $i=X, Y, Z$ axes, Figure 3 ). The correlation of three axial elastic moduli with BV/TV and pBV/TV had 

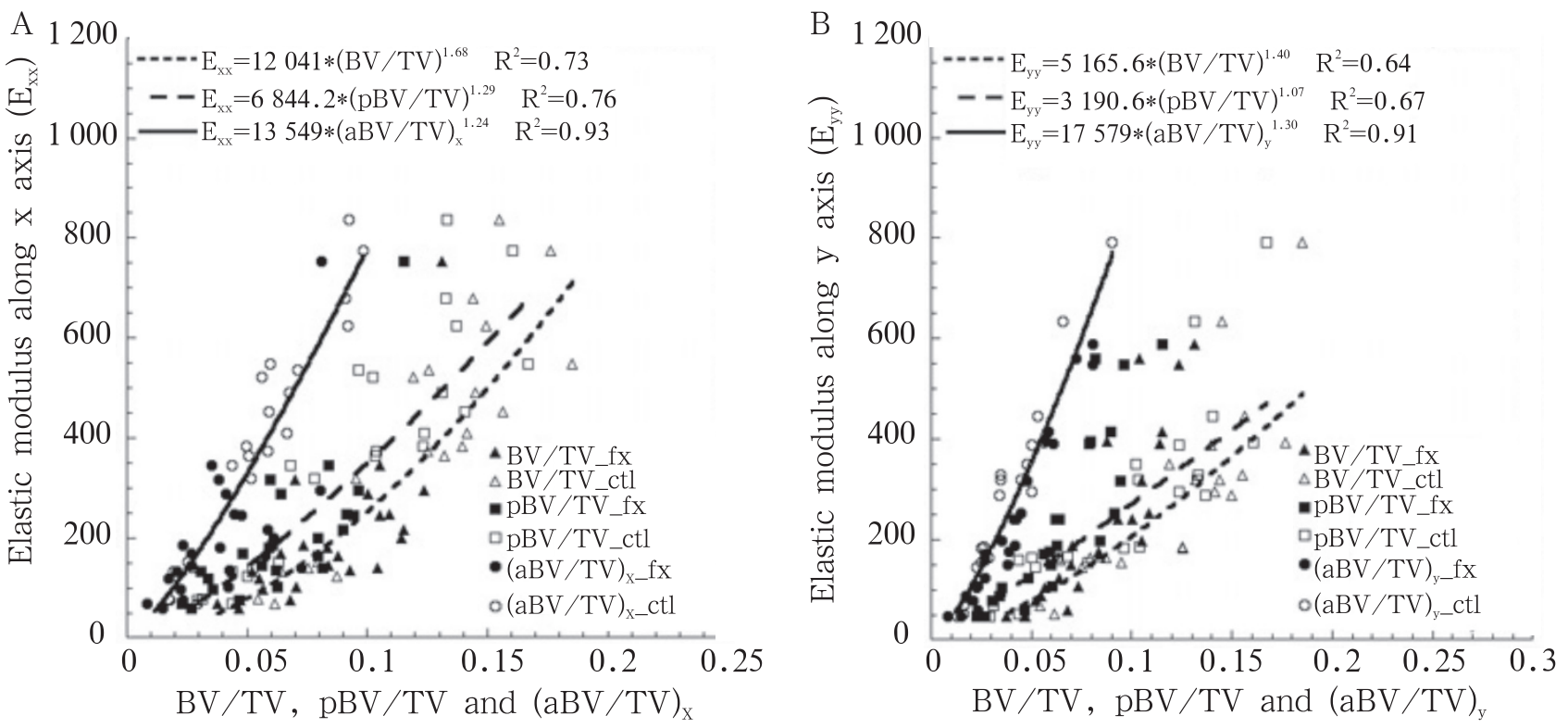

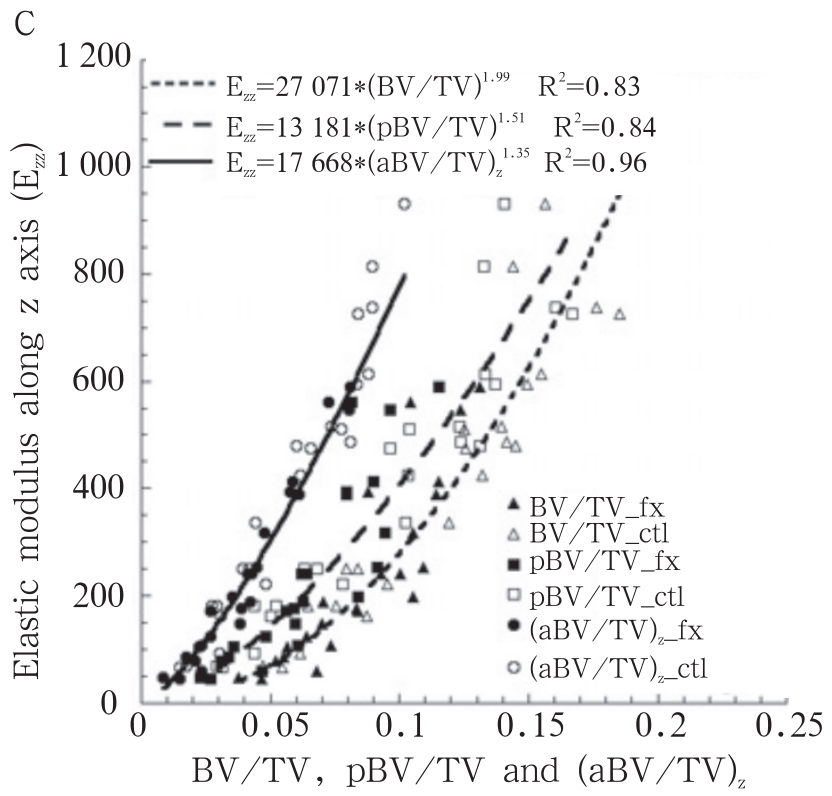

Figure 3 Correlations between axial elastic modulus $\mathrm{E}_{\mathrm{ii}}$ and bone volume fraction $(B V / T V)$, plate bone volume fraction $(p B V / T V)$ and axial bone volume fraction $\left(a B V / T V_{i}\right)$ by power law nonlinear regression $(i=X, Y, Z)$.

the same strength. Although axial bone volume only accounted for $30 \%-50 \%$ of the total bone volume, correlations between Eii and $a B V / T V i$ were generally stronger than those with $B V / T V$ and $p B V / T V$.

\section{Discussion}

In this study, we found that patients with intertrochanteric hip fractures had highly significant differences in trabecular microstructure and mechanical properties near the fracture site. Assessing trabecular bone micro- structure on the individual trabecula level by ITS demonstrated that bone loss and mechanical deficiency in hip fracture subjects were associated with lower $p B V / T V$, lower plate-rod ratio, decreased plate surface area and rod length, and a less axially aligned trabecular network, despite the increased connectivity between trabecular rods. Several studies used $\mathrm{MCT}$ standard morphological analyses to evaluate femoral neck microstructure in postmenopausal women with and without hip fractures. Consensus findings suggested that postmenopausal women with hip fractures had lower BV/TV, 
more widely-spaced and rod-like trabeculae, less connected and more anisotropic trabecular network at the femoral neck $(7,10-11)$. In present study, we further clarified the different pattems of bone loss in plate-like and rod-like trabeculae by characterizing the volume, morphology and orientation of individual trabecular platesand rods. We found preferential loss of trabecular plate bone volume and surface area in hip fracture subjects, whereas rod bone volume slightly increased and the number of rod-rod junctions increased significantly. Besides, striking differences were observed in the alignment of trabecular network along the longitudinal and transverse directions, as shown by dramatically reduced axial bone volume fraction along $X, Y$ and $Z$ axes. Our find ings suggested that conversion from platelike trabeculae to rod-like trabeculae and disrupted trabecular network orientations were two major characteristics of microstructure changes associated with hip fracture.

Previous studies using ITS a nalyses have demonstrated plate-like trabeculae to be the predominant contributor to mechanical competence of trabecular bone, and mechanical loading was mainly sustained by axially aligned trabeculae (17-18). In accordance with the observations from femoral neck, tibia and vertebral body, we also found that pBV/TV comelated with axial elastic moduli as strongly as the BV/TV of the intertrochanteric trabecular bone. Moreover, significantly stronger correlations were found between aBV/TV and corresponding axial elastic moduli along each of the $X$, $Y$ and $Z$ directions. Though the correlation coefficient between BV/TV (or pBV/TV) and $E_{i i}$ varied from 0.80 to $0.92(i=X, Y, Z)$, correlation coeffic ients between a BV/TV and $E_{i i}$ maintained as high as 0.95 to 0.98 , indicating aBV/TV as the most critical and robust determinant of the compressive mechanical properties. It has been dis covered that $C$ hinese-American women eamed greater bone strength than Caucasian-American women from vast advantages of more plate-like trabecular microstructure and much better aligned trabecular orientation, i.e. twice as high pBV/TV, 50\% higher a BV/TV (20). Echoing the study of Chinese vs. Caucasian bone quality, results in the present study proved that loss of trabecular plates and axially aligned trabeculae in the intertroc hanteric region signific antly diminished the resistance of femur to mechanical impact from various directions and likely led to fragility fractures at the hip.

Due to a series of mic rostructural defic its, patients who sustained hip fractures showed weakened whole femur strength and reduced failure load in sideway falls (26-27). Peak stress and strain distribution were mostly observed in the posterior aspect of the femoral neck and the intertrochanteric region by FE simula tion of a sideway fall that preceded most hip fractures (9). Concentrated peak strain within the trochanteric region was found to be highly predictive of trochanteric fractures, whereas uniform strain pattem in the femoral neck indicated femoral neck fractures (28). Our present study foc used on the subregion of intertrochanteric trabecular bone and provided more localized mechanistic insights into the changes of elastic moduli and shear moduli associated with hip fractures. Unlike the femoral neck trabecular network with a principal loading direction, trabecular bone in the intertrochanteric region subsisted under more complex loading conditions with mixed compressive and tensile stresses. Our FE results showed unifomly reduced axial elastic moduli and shearmoduli along the longitudinal and transverse directions, suggesting multidimensional impaiment of trabecular bone load-bearing capacity in the hip fracture patients.

Mic rostruc tural a lterations seen by ITS provided us new knowledge about pathogenesis of hip fracture in osteoporosis. Decrease of trabecular bone density and bone volume fraction largely resulted from diminished platelike trabeculae, known as the primary mechanical loading camier in trabecular network. The observation that rod-like trabeculae being well preserved, even slightly increased in fracture patients, might be explained by a combined effect of losing some rod-like trabeculae and regaining new rods from the remaining part of perforated trabecular plates (Figure 4). While the number and thickness of plate-like and rod-like trabeculae appeared unchanged, the size of individual plates and rods signific antly reduced, suggesting that the trabecular network became more fragmentary. More rod-like microstructure in the fracture group was evident by decreased plate-rod ratio, increased rod proportion and increased rod-rod connectivity. Since trabecular bone elastic modulus was detemined by plate bone volume through an approximately 1.5 power relation, the effect of plate tuming into rod on bone mechanical property was amplified into a marked increase of hip fracture risk.

Several limitations associated with this study need to be noted. First, due to relatively small sample size in this study, we did not separate male and female subjects for the ITS and $\mu \mathrm{FE}$ analyses. No significantly different outcomes were found from the two genders, so sex-pooled results were reported in this study. Additional studies of larger cohorts would be helpful to understand genderspecific characteristic s bone loss associated with hip fractures. Other limitations include those imposed by $\mu \mathrm{FE}$ analyses. Trabecular bone tissue was assumed to be constant in all specimens. Thus, the resulting mechanical 

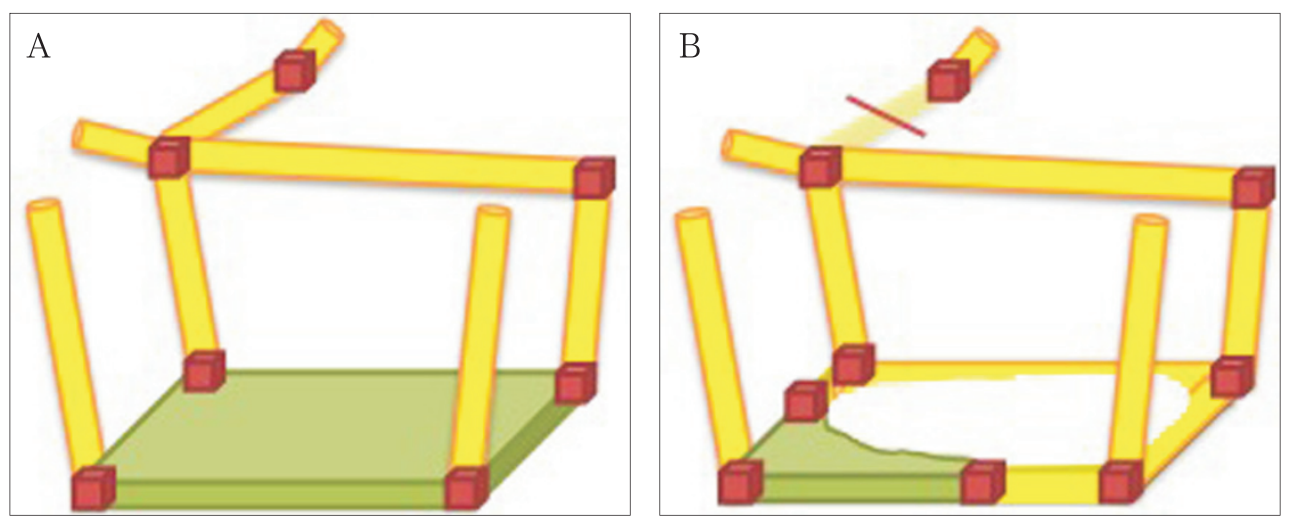

Figure 4 Possible mechanism of trabecular bone loss in hip fracture: perforated plate-like trabeculae turn into rods and residual small plate; broken rod-like trabeculae and new rods converted from plates result in unchanged overall trabecular rod volume.

measurements of $\mu \mathrm{FE}$ analysis reflected only the influence of microstructure but or intrinsic mineral quality. It remained unclear to what extent trabecular bone tissue property differed between the subjects with and without hip fractures. It would be of future interest to derive subject-specific tissue properties from high-resolution images and integrate them into $\mu \mathrm{FE}$ analyses.

In conclusion, ITS and FE analyses detected marked differences in trabecular microstructure and mechanical properties between patients with intertrochanteric fractures and nonfracture controls. Our findings evidenced conversion from plate-like to rod-like trabeculae and weakened trabecular network alignment in the intertrochanteric region adjacent to fracture site. Furthermore, decreased elastic moduli and shear moduli were observed in mechanically impaired fracture subjects. Overall, this report provided novel insights into the pattern of trabecular bone loss associated with intertrochanteric hip fractures and highlighted new biomechanical features in elderly individuals with intertrochanteric hip fractures.

\section{Acknowledgments}

The authors thank the mortuary staff and the staff of the Donor Tissue Bank of the Victorian Institute of Forensic Medicine for their assistance in the establishment of the Melbourne Femur Collection. They are also particularly grateful to the next-of-kin of the donors for their permission to remove material from the bodies for research purposes. This work was partially supported by grants from National Institutes of Health (AR051376, AR058004)

\section{References}

1 Burge R, Dawson-Hughes B, Solomon DH, Wong JB, King A,
Tosteson A. Incidence and economic burden of osteoporosisrelated fractures in the United States, 2005-2025. J Bone Miner Res. 2007;22:465-475

2 Riggs BL, Melton LJ 3rd. The worldwide problem of osteoporosis: insights afforded by epidemiology. Bone. 1995;17:505S-511S.

3 Gullberg B, Johnell O, Kanis JA. World-wide projections for hip fracture. Osteoporos Int. 1997;7:407-413.

4 Szulc P, Boutroy S, Vilayphiou N, Chaitou A, Delmas PD, Chapurlat R. Cross-sectional analysis of the association between fragility fractures and bone microarchitecture in older men: the STRAMBO study. J Bone Miner Res. 2011;26:1358-1367.

5 Wainwright SA, Marshall LM, Ensrud KE, Cauley JA, Black DM, Hillier TA, Hochberg MC, Vogt MT, Orwoll ES; Study of Osteoporotic Fractures Research Group. Hip fracture in women without osteoporosis. J Clin Endocrinol Metab. 2005;90:2787-2793.

6 Riggs BL, Melton Iii LJ 3rd, Robb RA, Camp JJ, Atkinson EJ, Peterson JM, Rouleau PA, McCollough CH, Bouxsein ML, Khosla S. Population-based study of age and sex differences in bone volumetric density, size, geometry, and structure at different skeletal sites. J Bone Miner Res. 2004;19:1945-1954.

7 Boutroy S, Vilayphiou N, Roux JP, Delmas PD, Blain H, Chapurlat $\mathrm{RD}$, Chavassieux P. Comparison of $2 \mathrm{D}$ and $3 \mathrm{D}$ bone microarchitecture evaluation at the femoral neck, among postmenopausal women with hip fracture or hip osteoarthritis. Bone. 2011;49:1055-1061.

8 Thomas CD, Mayhew PM, Power J, Poole KE, Loveridge N, Clement JG, Burgoyne CJ, Reeve J. Femoral neck trabecular bone: loss with aging and role in preventing fracture. J Bone Miner Res. 2009;24:1808-1818.

9 Carballido-Gamio J, Harnish R, Saeed I, Streeper T, Sigurdsson S, Amin S, Atkinson EJ, Therneau TM, Siggeirsdottir K, Cheng X, Melton LJ 3rd, Keyak J, Gudnason V, Khosla S, Harris TB, Lang TF. Proximal femoral density distribution and structure in relation to age and hip fracture risk in women. J Bone Miner Res. 2013;28:537-546.

10 Ciarelli TE, Fyhrie DP, Schaffler MB, Goldstein SA. Variations in 
three-dimensional cancellous bone architecture of the proximal femur in female hip fractures and in controls. J Bone Miner Res. 2000;15:32-40.

11 Milovanovic P, Djonic D, Marshall RP, Hahn M, Nikolic S, Zivkovic V, Amling M, Djuric M. Micro-structural basis for particular vulnerability of the superolateral neck trabecular bone in the postmenopausal women with hip fractures. Bone. 2012; 50:63-68.

12 Liu XS, Stein EM, Zhou B, Zhang CA, Nickolas TL, Cohen A, Thomas V, McMahon DJ, Cosman F, Nieves J, Shane E, Guo XE. Individual trabecula segmentation (ITS)-based morphological analyses and microfinite element analysis of HR-pQCT images discriminate postmenopausal fragility fractures independent of DXA measurements. J Bone Miner Res. 2012;27:263-272.

13 Cui WQ, Won YY, Baek MH, Lee DH, Chung YS, Hur JH, Ma YZ. Age-and region-dependent changes in three-dimensional microstructural properties of proximal femoral trabeculae. Osteoporos Int. 2008;19:1579-1587.

14 Singh M, Nagrath AR, Maini PS. Changes in trabecular pattern of the upper end of the femur as an index of osteoporosis. J Bone Joint Surg Am. 1970;52:457-467.

15 Li W, Kornak J, Harris T, Keyak J, Li C, Lu Y, Cheng X, Lang T. Identify fracture-critical regions inside the proximal femur using statistical parametric mapping. Bone. 2009;44:596-602.

16 Ding M, Hvid I. Quantification of age-related changes in the structure model type and trabecular thickness of human tibial cancellous bone. Bone. 2000;26:291-295

17 Liu XS, Cohen A, Shane E, Stein E, Rogers H, Kokolus SL, Yin PT, McMahon DJ, Lappe JM, Recker RR, Guo XE. Individual trabeculae segmentation (ITS)-based morphological analysis of highresolution peripheral quantitative computed tomography images detects abnormal trabecular plate and rod microarchitecture in premenopausal women with idiopathic osteoporosis. J Bone Miner Res. 2010;25:1496-1505

18 Liu XS, Sajda P, Saha PK, Wehrli FW, Bevill G, Keaveny TM, Guo XE. Complete volumetric decomposition of individual trabecular plates and rods and its morphological correlations with anisotropic elastic moduli in human trabecular bone. J Bone Miner Res.
2008;23:223-235.

19 Liu XS, Sajda P, Saha PK, Wehrli FW, Guo XE. Quantification of the roles of trabecular microarchitecture and trabecular type in determining the elastic modulus of human trabecular bone. J Bone Miner Res. 2006;21:1608-1617.

20 Liu XS, Walker MD, McMahon DJ, Udesky J, Liu G, Bilezikian JP, Guo XE. Better skeletal microstructure confers greater mechanical advantages in Chinese-American women versus white women. J Bone Miner Res. 2011;26:1783-1792.

21 Thomas C, Clement J. The Melbourne femur collection: How a forensic anthropological collection came to have broader applications//Stout S, Crowder C (eds.) Bone Histology: an Anthropological Perspective. CRC Press Inc, 2012

22 Saha PK, Chaudhuri BB, Majumder DD. A new shape preserving parallel thinning algorithm for 3D digital images. Pattern Recogn. 1997;30:1939-1955.

23 Saha PK, Chaudhuri BB. 3D digital topology under binary transformation with applications. Comput Vis Image Und. 1996;63: 418-429.

24 Guo XE, Goldstein SA. Is trabecular bone tissue different from cortical bone tissue? Forma. 1997;12:185-196

25 Hollister SJ, Brennan JM, Kikuchi N. A homogenization sampling procedure for calculating trabecular bone effective stiffness and tissue level stress. J Biomech. 1994;27:433-444.

26 Amin S, Kopperdhal DL, Melton LJ 3rd, Achenbach SJ, Therneau TM, Riggs BL, Keaveny TM, Khosla S. Association of hip strength estimates by finite-element analysis with fractures in women and men. J Bone Miner Res. 2011;26:1593-1600.

27 Dufour AB, Roberts B, Broe KE, Kiel DP, Bouxsein ML, Hannan MT. The factor-of-risk biomechanical approach predicts hip fracture in men and women: the Framingham Study. Osteoporos Int. 2012;23:513-520

28 Koivumaki JE, Thevenot J, Pulkkinen P, Salmi JA, Kuhn V, Lochmuller EM, Link TM, Eckstein F, Jamsa T. Does femoral strain distribution coincide with the occurrence of cervical versus trochanteric hip fractures? An experimental finite element study. Med Biol Eng Comput. 2010;48:711-717. 